\title{
Intestinal Perforation in Obstructed Umbilical Hernia due to Wedged Plum Seed
}

\author{
Rahul Gupta, ${ }^{1{ }^{*}}$ Vikram Singh Mujalde, ${ }^{1}$ Shilpi Gupta, ${ }^{1}$ Pradeep Kumar Gupta, ${ }^{1}$ Anu Bhandari, ${ }^{2}$ Praveen Mathur ${ }^{1}$
}

\begin{abstract}
The foreign body ingestion is a rare cause of gastrointestinal perforation in children and is typically seen with sharp foreign bodies or button batteries. Herein, we report an 11-month old male baby who presented with obstructed umbilical hernia. Abdominal radiograph showed dilated small bowel loops, while ultrasonography and CT scan suggested presence of a foreign body. Laparotomy revealed obstructed umbilical hernia with a plum seed being stuck in the terminal ileum causing intestinal perforation. Resection and anastomosis of intestine was performed.
\end{abstract}

Key words: Intestinal perforation; Plum seed; Obstructed hernia; Umbilical hernia

\section{INTRODUCTION}

The foreign body ingestion is a relatively common clinical problem in children. Most of them pass through the gastrointestinal tract spontaneously.[1,2] However, esophagus and small bowel are the most commonly involved sites of perforation and is typically seen with sharp foreign bodies or button batteries ingestion.[3,4] The presentation of foreign body as incarcerated umbilical hernia has been described in literature.[5] Herein, we report a case of perforation in obstructed umbilical hernia due to plum seed wedged in the terminal ileum.

\section{CASE REPORT}

An 11-month old male infant presented with bilious vomiting, abdominal distension and swelling in the umbilical region along with constipation for three days. Umbilical swelling was previously reducible. On examination, the patient was hemodynamically stable with mild dehydration, respiratory rate of $33 / \mathrm{min}$, and pulse rate of $120 / \mathrm{min}$. On physical examination, abdominal distention was present along with tender lump in the umbilical region suggestive of obstructed umbilical hernia. Laboratory investigations revealed total leucocyte count-20,600 mm3, hemoglobin-10.1 gm/dl, with normal renal and liver functions. Abdominal x-ray showed dilated small bowel loops (Fig.1); ultrasonography (USG) suggested $22 \mathrm{~mm}$ umbilical hernial defect with herniated bowel loops, small septate collection, along with a hyperechoic lesion with distal acoustic shadows in the umbilical region. CT scan demonstrated a $2.7 \mathrm{~cm}$ $x 1 \mathrm{~cm} \times 1 \mathrm{~cm}$ hyperdense lesion as content of the hernia (Fig.1).

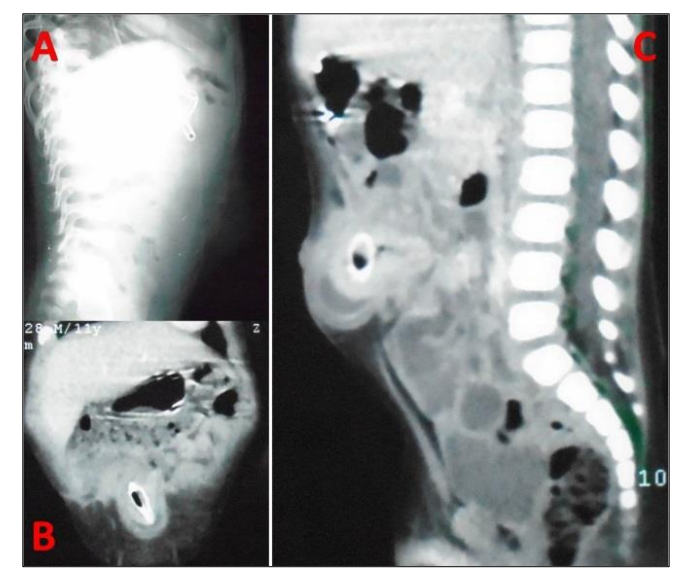

Figure 1: Preoperative radiograph showing a soft tissue shadow suggesting umbilical hernia (lateral view, A); Abdominal computed tomography scan images showing a foreign body as content of the umbilical hernia $(B, C)$.

Operation revealed obstructed umbilical hernia with terminal ileum $(1-2 \mathrm{cms}$ from ileo-cecal region) as its content (Fig. 2). Non-fixation of caecum and ascending colon was present. A plum seed which was wedged in the terminal ileum was seen to be coming out from the perforation in the bowel (Fig. 2). The seed was lying transversely resulting in two perforations in terminal ileum. The ileo-cecal region was markedly congested and edematous. Primary closure of gut was not feasible 
(Fig. 2). Resection of the ileo-cecum with ileoascending colon anastomosis was performed. Postoperative period was unremarkable, except for minor wound infection. Patient was started on oral feeds on the fifth postoperative day and remained well at follow up after discharge.

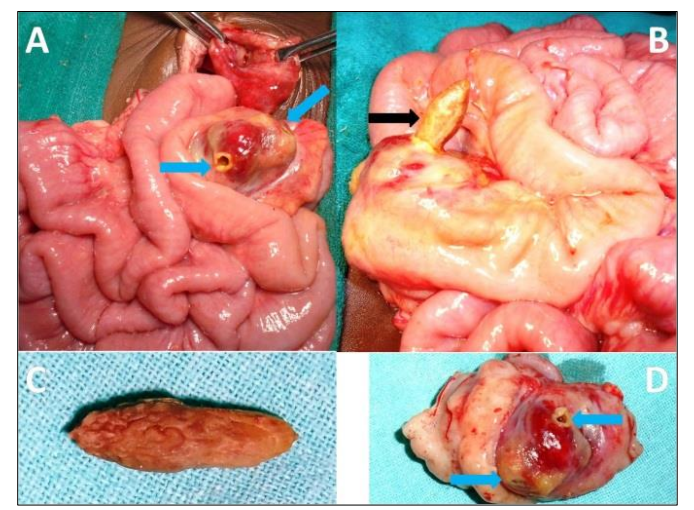

Figure 2: Per-operative photographs showing umbilical hernial sac, terminal ileum (1-2 cms from ileo-cecal region) incarcerated in umbilical hernia is seen with two perforations (diagonally opposite to each other as shown by blue arrows) due to wedged plum seed (A); Plum seed (black arrow) coming out through perforation (B); Plum seed (C) and resected specimen (D) with perforations (blue arrow).

\section{DISCUSSION}

Most of the ingested foreign bodies pass through the gastrointestinal tract (GIT) spontaneously. Few stuck $(<1 \%)$ in the anatomical transition regions (at the narrowing and angulations) of the GIT which include the upper, middle and lower esophagus, pylorus, ileocecal valve and rectosigmoid colon. Esophagus and small bowel are the most commonly locations involved.[3-5] Foreign bodies with sharp edges lead to serious complications (15\%-35\%) like intestinal obstruction, perforation and erosion into adjacent organs.[6] Apart from plum seed, apricots, persimmons, peanuts and nectarine have been reported to cause perforation.[7] It appears that in our case, presence of seed itself led to irreducibil- ity of the previously reducible umbilical hernia and also sharp ends of the seed, in addition to attempt by peristalsis led to traumatic perforation in bowel inside the obstructed umbilical hernia.

Perforations of the appendix and Meckel's diverticulum due to a seed ingestion have also been described.[8,9] $A$ rare case of incarcerated inguinal hernia due to ingested foreign body has also been reported.[10] Foreign body ingestion presenting as obstructed umbilical hernia is extremely rare.[5] Senior author (PM) encountered a similar case of obstructed umbilical hernia due to ingestion of turmeric stem in a 5-year-old boy.

\section{REFERENCES}

1. Dereci S, Koca T, Serdaroğlu F, Akçam M. Foreign body ingestion in children. Turk Pediatri Ars. 2015;50:234-40.

2. Schwartz GF, Polsky HS. Ingested foreign bodies of the gastrointestinal tract. Am Surg. 1976;42:236-8.

3. Gregori D, Scarinzi C, Morra B, Salerni L, Berchialla P, Snidero $S$, et al. Ingested foreign bodies causing complications and requiring hospitalization in European children: results from the ESFBI study. Pediatr Surg Int. 2010; 52:26-32.

4. Goh BK, Chow PK, Quah HM, Ong HS, Eu KW, Ooi LL, et al. Perforation of the gastrointestinal tract secondary to ingestion of foreign bodies. World J Surg. 2006;30:372-7.

5. Mirza B, Sheikh A. Open safety pin ingestion presenting as incarcerated umbilical hernia. APSP J Case Rep. 2011;2:25.

6. Stack LB, Munter DW. Foreign bodies in the gastrointestinal tract. Emerg Med Clin North Am.1996;14:493-521.

7. Atila K, Guler S, Bora S, Gulay H. An unusual cause of small bowel perforation: apricot pit. Turk J Traum Emerg Surg. 2011;17:286-8.

8. Hulme P. Foreign body causing perforation of the appendix in an African boy. Pan Afr Med J. 2010; 5:5.

9. Su CH, Lee JY, Chang YT. Perforation of Meckel's diverticulum by a peanut presenting as a mesentery abscess. Iran $\mathrm{J}$ Pediatr. 2012;23:223-5.

10. Salaman R, Foster M. Ingested foreign body presenting as an irreducible inguinal hernia in a baby. $\mathrm{J}$ Pediatr Surg. 1993;28:262-3.

AFFILIATION:

1 Department of Paediatric Surgery, SMS Medical College Jaipur, Rajasthan, India

2 Department of Radiodiagnosis, SMS Medical College Jaipur, Rajasthan, India

\section{CORRESPONDENCE:*}

Dr. Rahul Gupta, Assistant Professor, Department of Paediatric Surgery,

SMS Medical College, Jaipur, Rajasthan, India

Email: meetsurgeon007@yahoo.co.in

Received on: $25-04-2016$

Accepted on: 14-05-2016

Competing Interests: None declared

Source of Support: $\mathrm{Ni}$ 